\title{
Extended-Spectrum Beta-Lactamase Infections
}

\author{
Seema Chandra
}

Published online: 28 June 2013

(C) Springer Science+Business Media New York 2013

\begin{abstract}
Extended-spectrum beta-lactamases are enzymes produced by certain Gram-negative bacteria that confer significant resistance to conventional beta-lactam antibiotics. These enzymes are increasingly found worldwide in pathogenic bacteria. The following chapter will describe the enzymes and their clinical relevance with a brief discussion of current treatment options.
\end{abstract}

Keywords Extended-spectrum beta-lactamase (ESBL) . E. coli $\cdot$ Klebsiella $\cdot$ Antibiotic resistance

\section{Introduction}

As antibiotic resistance becomes more common year by year, the average hospital-based clinician needs to have some level of familiarity with patterns of resistance and commonly encountered pathogens. While resistant Grampositive organisms such as methicillin-resistant Staphylococcus aureus are frequently encountered in today's hospital, the increase in drug-resistant Gram-negative organisms is also of significant concern. This chapter will focus on the increasing problem of bacteria producing extended-spectrum beta-lactamases (ESBL).

Antibiotics which contain a beta-lactam ring as part of their structure, or generally categorized as beta-lactam antibiotics, include penicillins, cephalosporins, carbapenems, and monobactams [1]. Beta-lactamase production by Gram-negative bacteria is the most common form of resistance to this category of antibiotic, and the beta-lactamase

S. Chandra $(\bowtie)$

Baptist Hospital of Miami, Miami, FL, USA

e-mail: seemach@baptisthealth.net serves to break down the beta-lactam ring and render the antibiotic ineffective [2].

\section{Classification}

There are two classification schemes in use currently for the beta-lactamases; one assigns a group based on the molecular structure of the enzyme while the other focuses on the functional characteristics. The molecular classification system assigns a letter from $\mathrm{A}$ to $\mathrm{D}$ based on structure. In the functional classification scheme revised by Bush and Jacoby [3], there are three groups of betalactamases.

Group 1 consists of cephalosporinases. These enzymes generally have a higher affinity for cephalosporins than benzylpenicillin, and can exhibit some resistance to carbapenems when the enzymes is present in high levels. In general this group is resistant to inhibition by a beta-lactamase inhibitor such as clavulanate, and can act on cephamycins, which include cefoxitin and cefotetan. There is a subgroup 1e, or the extended-spectrum AmpC betalactamases, which also has activity against ceftazidime and other oxyimino-beta-lactams [3].

Group 2 consists of the serine beta-lactamases and is the largest group of beta-lactamases. This group has multiple subgroups based on affinity for various antibiotics and the ability of a beta-lactamase inhibitor to block activity of the enzyme. Subgroup 2 be contains the enzymes generally described as ESBL. The ESBLs have activity against penicillins, cephalosporins, including oxyimino-beta-lactams, and monobactam but this activity can be blocked by the presence of a beta-lactamase inhibitor [3]. There are other ESBLs in group 2 which also have expanded activity against higher generation cephalosporins but lack inhibition by 
beta-lactamase inhibitors. Group 2 also contains a subgroup of carbapenemases which have been linked to hospitalassociated outbreaks of multidrug-resistant infections [3].

Group 3 consists of metallo-beta-lactamases, which are structurally distinct and require a zinc ion for activity. These enzymes have activity against carbapenems [3].

\section{Distribution of ESBL Producing Organisms}

ESBLs are most commonly found in Escherichia coli, Klebsiella pneumoniae, and Klebsiella oxytoca, but have been reported throughout the Enterobacteriaceae family as well as in Acinetobacter baumannii and Pseudomonas aeruginosa [1]. ESBLs were first documented in Europe in the mid 1980s. An ESBL present in Klebsiella ozaenae conferring resistance to cefotaxime was reported in Germany in 1985 [4] and an outbreak of ESBL producing K. pneumoniae was reported in France in 1987 [5].

Since that time, ESBLs have been documented worldwide, although the prevalence varies by country. A study looking at the worldwide rate of ESBL detection in K. pneumoniae, E. coli, Proteus mirabilis, and Salmonella species in the late 1990 s found there were marked differences between the studied populations [6]. In Latin America, $45 \%$ of $K$. pneumoniae isolates were ESBLproducing, and $22 \%$ of European isolates were also found to express an ESBL phenotype. In comparison, the percentages were lower in the United States (8\%) and Canada (5\%). A similar trend was found with E. coli and $P$. mirabilis isolates [6].

A particular strain of E. coli, ST131, has been implicated recently in much of the multi-drug resistant $E$. coli infection in the United States [7]. This bacteria has been associated predominantly with a particular ESBL, CTX-M-15, and ESBL negative strains of this bacteria have also been associated with significant fluoroquinolone resistance. Based on their data collected throughout the United States in 2007, this study estimates that $69 \%$ of ESBL producing E. coli, $67 \%$ of fluoroquinolone-resistant $E$. coli, and $52 \%$ of multidrug resistant E. coli can be attributed to the ST131 strain [7].

\section{Risk Factors for Infection and Colonization}

Although these organisms were first reported with a nosocomial source of infection, community-acquired infection is a growing problem. A recent study completed in the United States in 2009 and 2010 looked at rates of either colonization or infection with ESBL-producing E. coli. They found that $37 \%$ of the patients had communityassociated infection with the majority of these being urinary tract infection [8•]. In this group of communityacquired infections, $54 \%$ were caused by the previously mentioned ST131 strain of E. coli. This study also identified several risk factors for healthcare-associated infections; these included cardiovascular disease, renal disease, dementia, solid organ malignancies, and hospitalization within the last year [8•].

A multinational analysis of ESBL producing Enterobacteriaceae published in 2009 evaluated six sites in Canada, Spain, Turkey, Israel and France. This group found a similar rate of community acquired infections, $\sim 34 \%$ [9]. In patients with community-acquired infections, the following risk factors were identified: male gender, age over 65 years, and recent use of cephalosporin antibiotics. Other implicated factors included bladder catheter within 3 months, recent use of any antibiotic, and functional dependence [9].

In all patients with ESBL-producing Enterobacteriaceae, significant risk was associated with admission from a longterm care facility, age over 65 years, male gender, recent hospitalization in the past 3 months, and recent use of any antibiotic [9]. The highest odds ratio was actually associated with admission from a long-term care facility [9].

In the hospital setting there have been many risk factors associated with the development of colonization or infection with an ESBL-producing organism. In general, there is an association with longer hospital stays, especially those involving indwelling devices and invasive procedures, and in very ill patients [10]. Exposure to antibiotics, particularly cephalosporins, but also fluoroquinolones, trimethoprimsulfamethoxazole, aminoglycosides, and metronidazole, has also been implicated [10].

A recent summary of health care-associated infections reported to the Center for Disease Control from 2009 to 2010 found that $29 \%$ of Klebsiella isolates had significant cephalosporin resistance (resistant to cefepime, cefotaxime, ceftazidime, and ceftriaxone), $13 \%$ were carbapenem-resistant, and $17 \%$ were characterized as multidrugresistant [11]. In E. coli isolates, $19 \%$ were resistant to the cephalosporins listed above, $42 \%$ were fluoroquinoloneresistant, $2 \%$ carbapenem-resistant, and $4 \%$ multidrugresistant [11].

A scoring model was developed in Italy to predict which patients were likely to have an ESBL-producing infection. A score of 3 was assigned for any of the following variables: hospitalization within the last 12 months or admission from another health-care facility. Scores of 2 were assigned for each of the following: Charlson comorbidity index $\geq 4$, previous therapy with beta-lactams and/or fluoroquinolones, history of urinary catheterization in the past 30 days, and age 70 years or greater [12•]. With a cutoff score of three or greater, the sensitivity was found to be high at $94 \%$ but with a specificity of only $41 \%$. Using a higher cutoff score of six improved the specificity to $94 \%$ 
but with a lower sensitivity of $55 \%$ [12•]. Depending on further validation of this and other prediction models, these may play a role in determining which patients are higher risk for harboring an ESBL-producing infection and eventually guiding empiric therapy.

\section{Outcomes in Patients with Infections Caused by ESBL- Producing Organisms}

There is a trend toward poorer outcomes including prolonged hospitalization and mortality in patients who acquire an ESBL-producing organism, particularly in the setting of bacteremia. A meta-analysis in 2007 reviewed sixteen studies, which ranged from 1996 to 2003 and covered 11 countries [13]. This meta-analysis found a relative risk of 1.85 for mortality associated with bacteremia with an ESBL-producing Enterobacteriaeae when compared to a non-ESBL producer, and this increased relative risk was noted to be statistically significant [13]. What was not clear, however, is the causality of this association, as not enough data was available to adjust this relative risk for patient comorbidities. Nevertheless, when the ten studies reported on delays in effective antimicrobial therapy, a pooled relative risk of 5.56 was found for a delay in therapy when the organism was ESBL-producing, thus suggesting that a delay in effective therapy may have a role in causing the noted increase in mortality [13]. A subsequent meta-analysis in 2012 of 32 studies found that after adjusting for confounders, there was still increased mortality of patients with ESBL-associated bacteremia with an odds ratio of 1.52 [14]. Bacteremia with an ESBL-producing organism has also been associated with prolonged hospitalization; one study found the excess length of stay to be nearly 7 days [15].

\section{Treatment of ESBL-Producing Organisms}

As noted above, delay in effective therapy appears to play a role in the increased mortality associated with ESBLpositive bacteremia. If an ESBL-producing organism is suspected or confirmed, consultation with an infectious disease specialist is recommended to appropriately guide initial and subsequent therapy.

While carbapenems are considered the standard treatment for serious infections caused by ESBL-producing organisms [1], the increasing detection of carbapenem resistance in health care-associated infections is of concern. Overuse of the carbapenems will likely lead to increased resistance of bacteria to this class of antibiotics. A systematic review and meta-analysis in 2012 looked at carbapenems and alternatives for the treatment of ESBLpositive bacteremia. The authors found that there was no statistical difference in mortality with either empiric or definitive therapy between carbapenems and beta-lactam/ beta-lactamase inhibitor (BL/BLI) combinations [16]. They do note that some caution should be used in interpreting this finding, as several of the patients who received empiric therapy with BL/BLIs were switched to carbapenem therapy as definitive therapy. They also found statistically increased mortality in patients treated with other antibiotics (neither carbapenems nor BL/BLIs) when compared with carbapenems. Therefore, while carbapenems remain the treatment of choice for ESBL-associated bacteremia, there may be a role for use of BL/BLIs in certain patients.

There may be a role for alternative antibiotics such as fosfomycin in the treatment of urinary tract infections caused by ESBL producing E. coli. An evaluation of the in vitro sensitivity of these organisms isolated from urine specimens in ambulatory patients found $97 \%$ were susceptible to fosfomycin [17]. However, data from the clinical world is limited. In a recent small retrospective analysis of patients receiving fosfomycin for treatment of urinary tract infections caused by multidrug-resistant organisms, the subgroup of patients with ESBL-producing organisms had a microbiologic cure rate of $57 \%$ [18]. Additionally, the IDSA guidelines on treatment for uncomplicated cystitis and pyelonephritis in women cite the lack of susceptibility testing for fosfomycin in most clinical laboratories as well the lack of data from randomized controlled studies as barriers to including fosfomycin in their recommendations. They do note that "observational studies are supportive of clinical efficacy" [19].

The most recent IDSA guideline for treatment of hospital-acquired pneumonia was published in 2005 and is currently being revised [20]. Nevertheless, this guidelines recommends that if ESBL-producing $K$. pneumoniae is suspected then initial treatment with a carbapenem is a "reliable choice" [20].

\section{Summary}

As the problem of antibiotic resistance increases, hospitalbased health-care providers will be faced with difficult antibiotic management decisions more frequently than ever before. Every clinician caring for hospitalized patients needs to be aware of the common resistant organisms encountered both in the hospital and the community, including the ESBL producers. More study is certainly required in risk prediction models to assist in gauging which patients are at high-risk for infection with an ESBLproducing infection. 
While overuse of antibiotics such as carbapenems is likely contributing to increasing resistance, there is also data that mortality increases significantly if there is a delay in treatment for patients with serious infections caused by ESBL-producing Enterobacteriaceae. At this time, carbapenems remain the first-line of therapy for these infections, with a possible role for alternate agents such as BL/BLIs and fosfomycin and others in certain subtypes of infections. To guide therapy as well as the use of possible alternate agents, an infectious diseases specialist should be involved when possible in the initial and subsequent treatment of patients at a high risk for or confirmed to have infection with an ESBL-producing organism.

Disclosure No potential conflicts of interest relevant to this article were reported. This article does not contain any studies with human or animal subjects performed by any of the authors.

\section{References}

Papers of of particular interest, published recently, have been highlighted as:

- Of importance

1. Jacoby GA, Munoz-Price LS. The new $\beta$-lactamases. N Engl J Med. 2005;352:380-91.

2. Chambers HF. Penicillins and $\beta$-lactam inhibitors. In: Mandell GL, Bennett JE, Dolin R, editors. Mandell, Douglas and Bennett's principles and practice of infectious diseases, 7th ed. Philadelphia: Elsevier; 2010. p. 309-322.

3. Bush K, Jacoby GA. Updated functional classification of $\beta$-lactamases. Antimicrob Agents Chemother. 2010;54(3):969-76.

4. Kliebe C, Nies BA, Meyer JF, et al. Evolution of plasmid-coded resistance to broad-spectrum cephalosporins. Antimicrob Agents Chemother. 1985;28(2):302-7.

5. Brun-Buisson C, Legrand P, Philippon A, et al. Transferable enzymatic resistance to third-generation cephalosporins during nosocomial outbreak of multiresistant Klebsiella pneumoniae. Lancet. 1987;2(8554):302-6.

6. Winokur PL, Canton R, Casellas JM, Legakis N. Variation in the prevalence of strains expressing an extended-spectrum beta-lactamase phenotype and characterization of isolates from Europe, the Americas, and the Western Pacific region. Clin Infect Dis. 2001;32(Suppl 2):S94-103.

7. Johnson JR, Johnston B, Clabots C, et al. Escherichia coli sequence type ST131 as the major cause of serious multidrugresistant $E$ coli infections in the United States. Clin Infect Dis. 2010;51(3):286-94.

8. - Doi Y, Park YS, Rivera JI, et al. Community-associated extended-spectrum $\beta$-lactamase-producing Escherichia coli infection in the United States. Clin Infect Dis. 2013;56(5): 641-8. This is a recent update on the spectrum of community-acquired
ESBL infections in the United States with an assessment of risk factors for acquisition of these organisms.

9. Ben-Ami R, Rodriguez-Bano J, Arslan H, et al. A multinational study of risk factors for infection with extended-spectrum $\beta$-lactamase-producing Enterobacteriaceae in nonhospitalized patients. Clin Infect Dis. 2009;49:682-90.

10. Patterson DL, Bonomo RA. Extended-spectrum $\beta$-lactamases: a clinical update. Clin Microbiol Rev. 2005;18(4):657-86.

11. Sievert DM, Ricks P, Edwards JR, et al. Antimicrobial-resistant pathogens associated with healthcare-associated infections: summary of data reported to the National Healthcare Safety Network at the Centers for Disease Control and Prevention, 2009-2010. Infect Control Hosp Epidemiol. 2013;34(1):1-14.

12. - Tumbarello M, Trecarichi EM, Bassetti M, et al. Identifying patients harboring extended-spectrum-beta-lactamase-producing Enterobacteriaceae on hospital admission: derivation and validation of a scoring system. Antimicrob Agents Chemother. 2011;55(7):3485-90. This is an attempt to generate a prediction system to enable the front-line clinician to estimate the chance of a particular patient having an ESBL-producing organism. These types of tools will become increasingly clinically relevant as they are refined and validated in additional populations.

13. Schwaber MJ, Carmeli Y. Mortality and delay in effective therapy associated with extended-spectrum $\beta$-lactamase production in Enterobacteriaceae bacteraemia: a systematic review and metaanalysis. J Antimicrob Chemother. 2007;60(5):913-20.

14. Rottier WC, Ammerlaan HS, Bonten MJ. Effects of confounders and intermediates on the association of bacteraemia caused by extended-spectrum $\beta$-lactamase-producing Enterobacteriaceae and patient outcome: a meta-analysis. J Antimicrob Chemother. 2012;67(6):1311-20.

15. Stewardson A, Fankhauser C, De Angelis G, et al. Burden of bloodstream infection caused by extended-spectrum $\beta$-lactamaseproducing Enterobacteriaceae determined using multistate modeling at a Swiss University Hospital and a nationwide predictive model. Infect Control Hosp Epidemiol. 2013;34(2):133-43.

16. Vardakas KZ, Tansarli GS, Rafailidis PI, et al. Carbapenems versus alternative antibiotics for the treatment of bacteraemia due to Enterobacteriaceae producing extended-spectrum $\beta$-lactamases: a systematic review and meta-analysis. J Antimicrob Chemother. 2012;67(12):2793-803.

17. Auer S, Wojna A, Hell M. Oral treatment options for ambulatory patients with urinary tract infections caused by extended-spectrum-beta-lactamase-producing Escherichia coli. Antimicrob Agents Chemother. 2010;54(9):4006-8.

18. Neuner EA, Sekeres J, Hall GS, et al. Experience with fosfomycin for treatment of urinary tract infections due to multidrug-resistant organisms. Antimicrob Agents Chemother. 2012;56(11):5744-8.

19. Gupta K, Hooton TM, Naber KG, et al. International clinical practice guidelines for the treatment of acute uncomplicated cystitis and pyelonephritis in women: a 2010 update by the Infectious Diseases Society of America and the European Society for Microbiology and Infectious Diseases. Clin Infect Dis. 2011;52(5):e103-20.

20. American Thoracic Society, Infectious Diseases Society of America. Guidelines for the management of adults with hospitalacquired, ventilator-associated, and healthcare-associated pneumonia. Am J Respir Crit Care Med. 2005;171:388-416. 